\title{
Radiant Heat Transfer in Packed Beds
}

\author{
JOHN C. CHEN and STUART W. CHURCHILL
}

University of Michigan, Ann Arbor, Michigan

\begin{abstract}
The effective scattering and absorption cross sections were determined from measurements of radiant transmission through isothermal beds of glass, aluminum oxide, steel and silicon carbide spheres, cylinders, and irregular grains. The radiant conductivities were calculated from these cross sections. Back scattering was found to be the principal mechanism of attenuation for all of the packings. Absorption was a significant mechanism only for the silicon carbide grains. At $2,000^{\circ} \mathrm{F}$. the radiant contribution to the total heat flux was estimated to vary from $35 \%$ for the silicon carbide packings to $85 \%$ for the glass packings.
\end{abstract}

Radiation is known to be an important mechanism of heat transfer in packed beds, particularly at high temperatures. The many models which have been proposed to describe simultaneous radiation and conduction may be classified in three groups.

The first type is based on those idealized geometrical arrangements of the phases which permit direct algebraic formulation of the transfer processes. Nusselt (1), Damköhler (2), and Argo and Smith (3) all treated the solid and gas phases as alternating layers perpendicular to the direction of transfer. If the distance between layers of solid is assumed to be 1 particle diameter, the following expression is obtained for the radiant conductivity of the gas phase:

$$
k_{r}^{g}=4 \sigma D \epsilon T^{3} /(2-\epsilon)
$$

The radiant conductivity is defined as the radiant heat flux density divided by the temperature gradient. Schotte (4) considered a lattice of particles with radiation and conduction in parallel and obtained the following expression:

$$
k_{r}=\frac{1-\delta}{\frac{1}{k_{o}{ }^{g}}+\frac{1}{k_{r}^{g}}}+\delta k_{r}^{g}
$$

with

$$
k_{r}^{g}=4 \sigma \in D T^{3}
$$

Laubitz (5), Yagi and Kunii (6), and Hill and Wilhelm (7) also developed models of this type.

J. C. Chen is with Brookhaven National Laboratory, Upton, New York
The second type is based on a random walk process. Rosseland (8) interpreted the radiant transfer in terms of the diffusion of photons and obtained the following expression for the radiant conductivity:

$$
k_{r}=4 \sigma l T^{3} / 3
$$

Bosworth (9) suggested that for a packed bed the mean free path should be set equal to the particle diameter.

In the third type of model the packed bed is considered to be a pseudohomogeneous material, permitting description of the heat transfer processes by differential or integrodifferential equations and boundary conditions. Van der Held (10) added a radiant transfer term to the Fourier equation for conduction and obtained the following expression for the radiant conductivity far away from the boundaries of the packing:

$$
k_{r}=16 \sigma n^{2} T^{3} / 3(a+s)
$$

Hamaker (11) suggested a two-flux representation of radiant transfer leading to three coupled, differential equations for radiation and conduction. He obtained general solutions in terms of arbitrary boundary constants but did not present specific results for a finite bed. This solution is utilized and extended in this paper.

The data for heat transfer in heterogeneous materials have recently been reviewed by Gorring and Churchill (12). Data for the radiant contribution are very limited. Larkin and Churchill (13) made direct measurements of radiant transfer in fibrous and foamed insulations of high porosity. Apparently no comparable measurements have been made for packed beds. Hill and Wilhelm (7) measured the total heat transfer rate in a bed of alumina spheres at temperatures up to $1,000^{\circ} \mathrm{C}$. and estimated the radiant contribution by subtracting the rate of conduction as estimated from the correlation of Wilhelm, Johnson, Wynkoop, and Collier (14). Schotte (4) analyzed the data of Yagi and Kunii (6) for total transfer in packed beds and concluded that the radiant contribution was in some cases as high as $80 \%$.

The objective of this investigation was to measure directly the contribution of radiation to heat transfer in packed beds. Variables considered were the size and shape of packing, packing material, porosity, and temperature.

\section{THEORETICAL MODEL}

The two-flux model formulated by Hamaker (11) and utilized by Larkin and Churchill (13) was used as a basis for this investigation. Representation of the radiant intensity in the packed media by two discrete fluxes, one forward and one backward, permits reduction of the general integro-differential equation for radiant transport to the following two differential equations:

$$
\frac{d i}{d x}=-(a+b) i+b j+a \sigma T^{4}
$$




$$
-\frac{d j}{d x}=-(a+b) j+b i+a \sigma T^{4}
$$

The first term on the right-hand side of Equation (6) represents the decrease in the forward component of the radiant flux by absorption and scattering; the second term represents the increase due to scattering of the backward flux into the forward direction, and the third term represents radiant emission from the solid into the forward direction. Equation (7) parallel planes with temperatures $T_{1}$ and $T_{2}$ and emissivities $\epsilon_{1}$ and $\epsilon_{2}$ are

$$
\begin{aligned}
& T=T_{1} \text { and } i=\epsilon_{1} \sigma T_{1}{ }^{4}+ \\
& \left(1-\epsilon_{1}\right) j \text { at } x=0 \\
& T=T_{2} \text { and } j=\epsilon_{2} \sigma T_{2}{ }^{4}+ \\
& \left(1-\epsilon_{2}\right) i \text { at } x=L
\end{aligned}
$$

The system of Equations (6) to (8) is nonlinear, and an exact solution has not been found. Larkin and Churchill (13) assumed the temperature to be a linear function of distance and obtained a solution which appears to be

$$
\begin{array}{r}
\frac{q_{r}}{\sigma T_{1}{ }^{4}}=2 \sqrt{\frac{1+\gamma}{1+\frac{2 b}{a}} A[\gamma C-} \\
\frac{\left.e^{a X}-B e^{-a X}\right]}{k_{c}}=\frac{\gamma C-e^{a x}-B e^{-a X}}{C+e^{a X}+B e^{-a X}}
\end{array}
$$

where

$$
\begin{aligned}
& X=x / L \\
& \alpha=L \sqrt{a(a+2 b)(\gamma+1)} \\
& \gamma=8 \sigma T_{a}^{3} / k_{c}(a+2 b)
\end{aligned}
$$

can be interpreted similarly. A steady state energy balance for a differential volume yields the following relationship between the temperature and the radiant fluxes if natural convection is neglected:

$$
k_{\circ} \frac{d^{2} T}{d x^{2}}+a(i+j)-2 a \sigma T^{4}=0
$$

The corresponding heat flux densities for conduction and radiation are

$$
q_{\mathrm{e}}=-k_{\mathrm{o}} \frac{d T}{d x}
$$

and

$$
q_{r}=i-j
$$

The radiant conductivity is by definition

$$
k_{r}=-(i-j) /(d T / d x)
$$

The boundary conditions for heat transfer in a packed bed between two

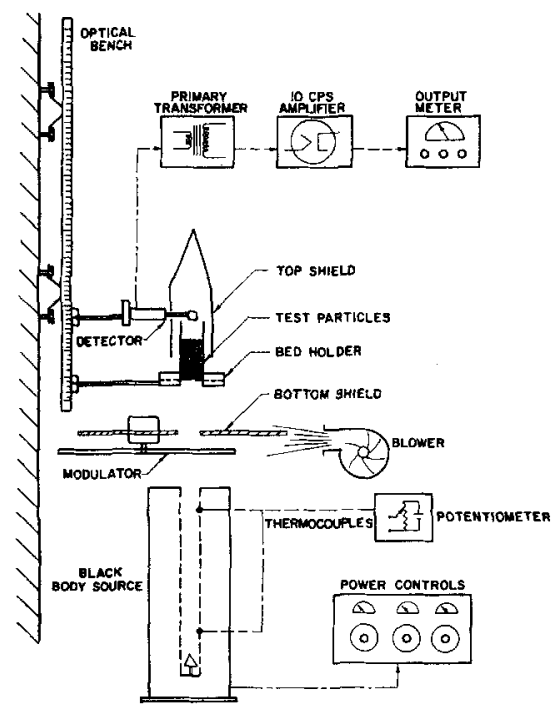

Fig. 1. Diagram of apparatus.

$$
B=\frac{e^{\alpha}\left[\left(\frac{\epsilon_{2}}{2-\epsilon_{2}}\right)\left(\frac{\gamma+1}{\alpha}\right)(a+2 b) L+1\right]}{\left(\frac{\epsilon_{1}}{2-\epsilon_{1}}\right)\left(\frac{\gamma+1}{\alpha}\right)(a+2 b) L+1}
$$

a reasonable approximation for many practical cases. Hamaker (11) developed a solution for small temperature differences by approximating $T^{4}$ by the first two terms of a Taylor's series expansion about some mean temperature $T_{o}$. This solution is completed for boundary conditions (12) and (1) in reference 15 . The resulting expressions for the heat flux densities and radiant conductivity are

$$
\begin{gathered}
\frac{q_{0}}{\sigma T_{2}{ }^{4}}=2 \sqrt{\frac{1+\gamma}{1+\frac{2 b}{a}}} \cdot A[C+ \\
\left.e^{a x}+B e^{-a x}\right]
\end{gathered}
$$

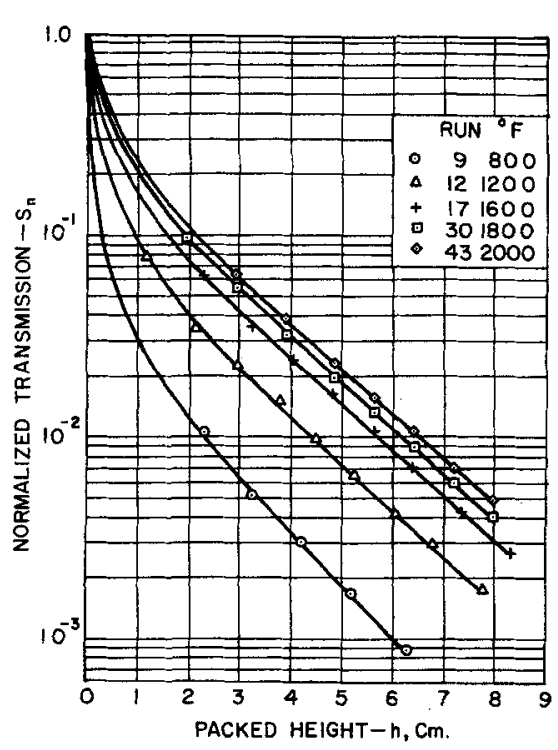

Fig. 2. Transmission data for packed beds of 5-mm glass spheres (GS-5).

$$
\begin{array}{r}
C=\frac{1}{\alpha}\left[\frac{\xi}{A \gamma}-\left(e^{a}-1\right)-\right. \\
\left.B\left(1-e^{-a}\right)\right]
\end{array}
$$

and

$$
\xi=\left(T_{1}^{4}-T_{2}^{4}\right) / T_{1}^{4}
$$

From Equation (16) it is apparent that the radiant conductivity is a function of position, bed thickness, and the boundary emissivities and temperatures, as well as of the bed properties. However for locations sufficiently far from the boundaries of an optically thick bed such that $\alpha X>>1$ and $\alpha(1-X)>>1$, Equation (16) approaches

$$
k_{r}=\frac{8 \sigma T_{o}{ }^{\mathbf{3}}}{a+2 b}
$$

This relationship was also obtained by Hamaker (11) by a different procedure.

If absorption is negligible $(a=0)$, Equations (6), (7), and (8) simplify greatly, and the following exact solution is obtained:

$$
\begin{gathered}
T=T_{1}-\left(T_{1}-T_{2}\right)(x / L) \\
q_{c}=k_{c}\left(T_{1}-T_{2}\right) / L \\
q_{r}=\frac{\sigma\left(T_{1}^{4}-T_{2}^{4}\right)}{\frac{1}{\epsilon_{1}}+\frac{1}{\epsilon_{2}}-1+b L} \quad(27) \\
k_{r}=\frac{\sigma\left(T_{1}^{4}-T_{2}^{4}\right) L}{\left(\frac{1}{\epsilon_{1}}+\frac{1}{\epsilon_{2}}-1+b L\right)\left(T_{1}-T_{2}\right)}
\end{gathered}
$$




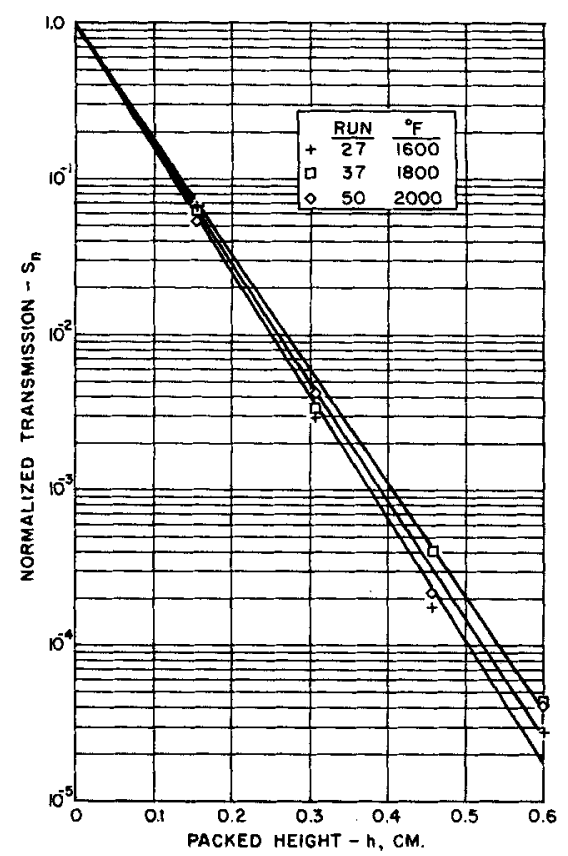

Fig. 3. Tronsmission dato for packed beds of 16-mesh silicon carbide grains (CG-16).

For large $L$ Equation (28) becomes equivalent to Equation (24) for $a=0$.

Radiant heat transfer can be computed in any packed bed if $k_{r}$ is known. The expressions which were derived above relate $k_{r}$ to the cross sections $a$ and $b$. The balance of this investigation is concerned with the experimental determination of $a$ and $b$ for some typical packings.

\section{EXPERIMENTAL MODEL}

The experimental work was based on the blackbody transmission tech- nique used by Larkin and Churchill (13). A variable-temperature source provided a flux of blackbody radiation on one surface of a bed of packing. The radiant flux from the source was modulated by mechanical chopping to approximate a 10 cycle/sec. square wave. The intensity of the flux transmitted through various depths of packing was measured with a thermopile detector. The detector signal was passed through a primary transformer to eliminate the direct current portion of the voltage and then to a narrowband amplifier which was finely tuned to amplify only $10 \mathrm{cycle} / \mathrm{sec}$. signals. Thus the output of the amplifier was proportional solely to scattered plus directly transmitted radiation from the

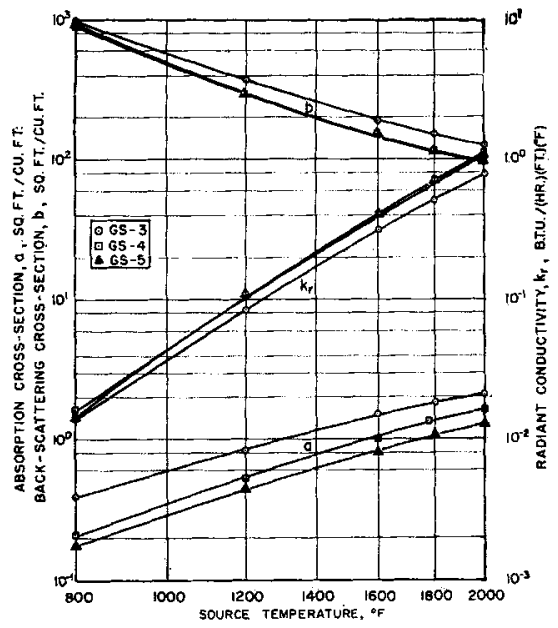

Fig. 4. Radiant properties of packed beds of glass spheres.

$$
S_{n}=\frac{i(h)}{i_{o}}=\frac{1}{\cosh [\sqrt{a(a+2 b)} h]+\frac{a+b}{\sqrt{a(a+2 b)}} \sinh [\sqrt{a(a+2 b)} h]}
$$

source. For this case Equations (6) and (7) degenerate to

$$
\begin{aligned}
& \frac{d i}{d x}=-(a+b) i+b j \\
& -\frac{d j}{d x}=-(a+b) j+b i
\end{aligned}
$$

and for totally absorbing boundaries the boundary conditions become

$$
\begin{aligned}
& i=i_{a} \text { at } x=0 \\
& i=0 \text { at } x=h
\end{aligned}
$$

This problem can be solved to yield the following expression for the normalized transmission:
Equation (33) defines the absorption and scattering cross sections $a$ and $b$ in terms of the transmission and the height of packing and was used to correlate the experimental data.

\section{EXPERIMENTAL APPARATUS AND PROCEDURE}

The arrangement of the experimental equipment is shown in Figure 1 . The blackbody source was an open-end tubular furnace. The core was a silicon carbide tube 3 in. in diameter and 27 in. long, closed at one end. Three electric heating coils were wrapped around the tube to control the temperature of the core. The inside-wall temperature was measured with

Table 1. Description and Properties of Packinc

$$
\begin{array}{ll|l}
\text { Properties* } & \\
k_{s}, & \text { Packings }
\end{array}
$$$$
T,{ }^{\circ} \mathrm{F} . \quad \text { e } \frac{\text { B.t.u. }}{(\mathrm{hr} .)(\mathrm{ft} .)\left({ }^{\circ} \mathrm{F} .\right)} \quad \text { Code Shape }
$$

\begin{tabular}{lrrr}
\hline Boro-silicate glass & 800 & 0.9 & 0.63 \\
& 1,200 & 0.8 & 0.63 \\
& 1,600 & & 0.63 \\
& 1,800 & & 0.63 \\
& 2,000 & & 0.63 \\
\hline
\end{tabular}

$\begin{array}{llll}\text { Aluminum oxide } & 1,600 & 0.32 & 2.22 \\ & 1,800 & 0.28 & 2.29 \\ & 2,000 & 0.28 & 2.36\end{array}$

\begin{tabular}{llll}
\hline Polished carbon steel & 1,600 & 0.4 & 17 \\
& 1,800 & 0.4 & 16 \\
& 2,000 & 0.4 & 15 \\
\hline Silicon carbide & 1,600 & 0.88 & 8.75 \\
& 1,800 & 0.91 & 8.25 \\
& 2,000 & 0.92 & 7.75
\end{tabular}

$\begin{array}{ll}\text { GS-3 } & \text { spheres } \\ \text { GS-4 } & \text { spheres } \\ \text { GS-5 } & \text { spheres }\end{array}$$$
D
$$$$
8
$$

\begin{tabular}{llll}
\hline AS-3/16 & spheres & $3 / 16 \mathrm{in}$. & 0.49 \\
AP-1/8 & cylindrical pellets & $1 / 8 \times 1 / 8 \mathrm{in}$. & 0.66 \\
AP-5/32 & cylindrical pellets & $5 / 32 \times 5 / 32$ in. & 0.69 \\
AP-3/16 & cylindrical pellets & $3 / 16 \times 3 / 16 \mathrm{in}$. & 0.67 \\
AG-4 & irregular grains & $6.6 \mathrm{~mm}$. & 0.61 \\
AG-16 & irregular grains & $1.7 \mathrm{~mm}$. & 0.54 \\
\hline SS-1/8 & spheres & $1 / 8 \mathrm{in}$. & 0.39 \\
SS-3/16 & spheres & $3 / 16 \mathrm{in}$. & 0.40 \\
& & &
\end{tabular}

$\begin{array}{lll}\text { CG-16 irregular grains } & 1.7 \mathrm{~mm} . & 0.48\end{array}$ 


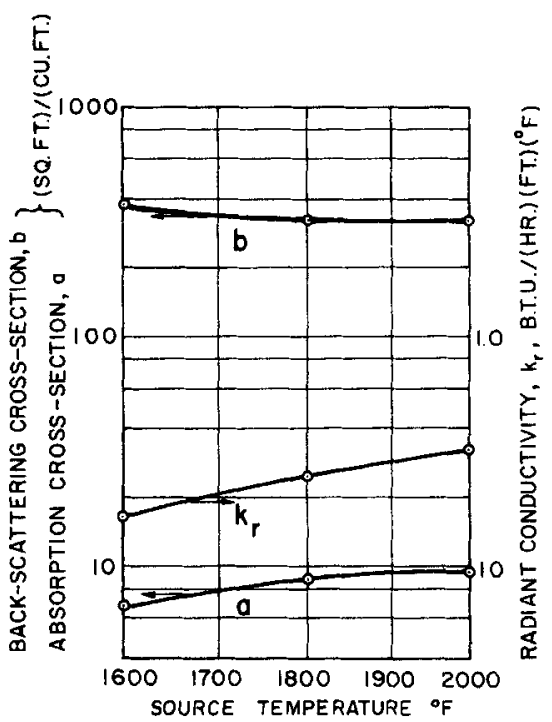

Fig. 5. Radiant properties of packed beds of 3/16-in. steel spheres (SS-3/16).

chromel-alumel thermocouples 3 and 24 in. from the top. The temperature differences between these points were kept within $5^{\circ} \mathrm{F}$. A reflector cone of aluminum oxide $2 \frac{1}{2}$ in. in diameter was positioned 2 in. above the bottom of the core to shield the bottom and increase the emissivity of the enclosure. Calculations based on an emissivity of 0.9 for the silicon carbide indicate that the effective emissivity of the source was greater than 0.99 .

Air was circulated across the space between the source and the test bed to prevent convective heating of the bed and to cool the chopper and shields. Wall and control panels around the equipment were painted a dull black to minimize reflected radiation. In addition a bottom shield with a 21/2-in. hole, centered over the source, was placed above the modulator, and a conical shield was fitted over the bed and detector.

The detector was the same one used by Larkin and Churchill. The sensing element consisted of bismuth-antimony-tellerium thermocouples with a gold-black filmed junction. The sensing junction had a total cross section of approximately 0.1 by 0.15 in. The element was mounted in an evacuated brass case with a potassium bromide window. The output of the amplifier was measured by a milliammeter with a variable range. By use of a calibrated attenuator in the amplifier circuit and the dual range of the meter it was possible to maintain a precision of $\pm 2 \%$ for almost all readings. The linearity of the detector and amplifier circuit was verified by measuring the intensity from an effective point source as a function of distance. The signal from the amplifier was also found to vary linearly with the fourth power of the source temperature, thereby indicating the independence of the signal from the spectral distribution of the source.

The packed bed was designed to approximate a one-dimensional system. The actual bed was contained in a 2 -in. I.D., highly polished, aluminum tube. In order to test the validity of the simulation of an infinite horizontal layer of packing by a finite layer and highly reflecting walls, the

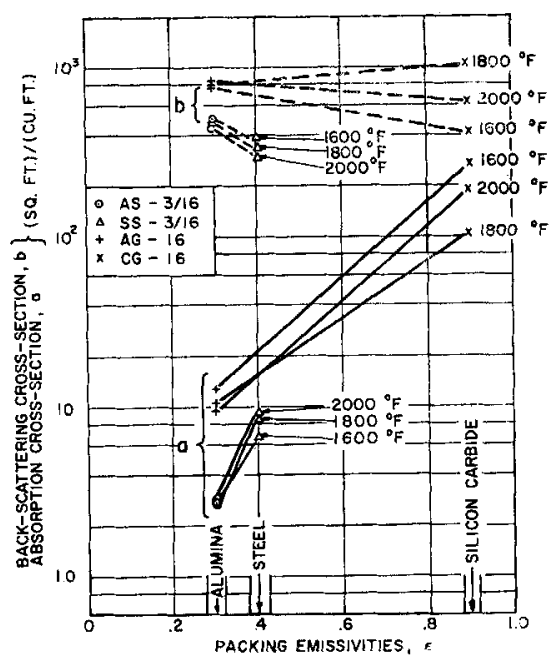

Fig. 6. Effect of emissivity on radiant properties of packed-beds.

length of the retaining tube was varied for fixed depths of packing. The resulting transmissions were virtually indistinguishable. The change in transmission was also negligible when the detector was moved laterally across the packing. This is an even stronger confirmation of the one dimensionality of the experimental system, since either absorption by the tube wall or nonuniform irradiation of the bed would have produced a change.

The test particles were supported on a screen. The experimental transmissions were normalized with respect to the retaining screen by making measurements without any packing for a variety of temperatures and tube lengths. Both 18 and 30 NBS-mesh screens were used. Slightly lower normalized transmissions were observed with the 30-mesh screen indicating

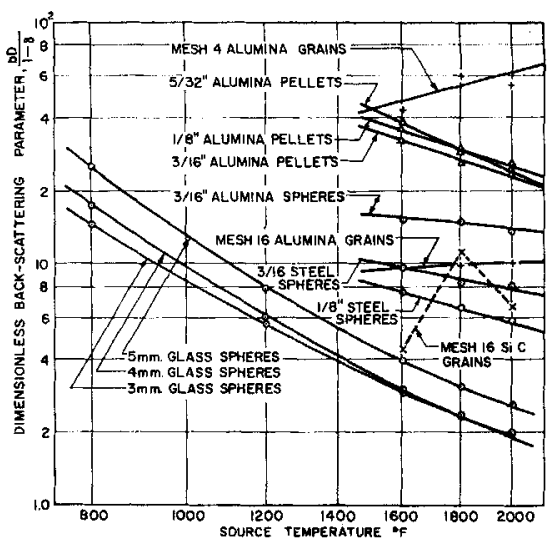

Fig. 7. Back scattering parameter for packed beds.

the normalization did not cancel out the effect of the screen completely. The corresponding difference in the radiant conductivity was about $7.6 \%$, indicating a possible uncertainty in the experimental results of this order.

Periodic repetition of the experiments confirmed their reproducibility. Further details concerning the experimental work are given in reference 15 .

\section{EXPERIMENTAL RESULTS}

A total of fifty-two runs were carried out with packings of twelve different particles. The particles and packings are described and coded in Table 1 . For the glass particles measurements were possible with source temperatures as low as $800^{\circ} \mathrm{F}$. For the more opaque particles accurate measurements were possible only at tempera-

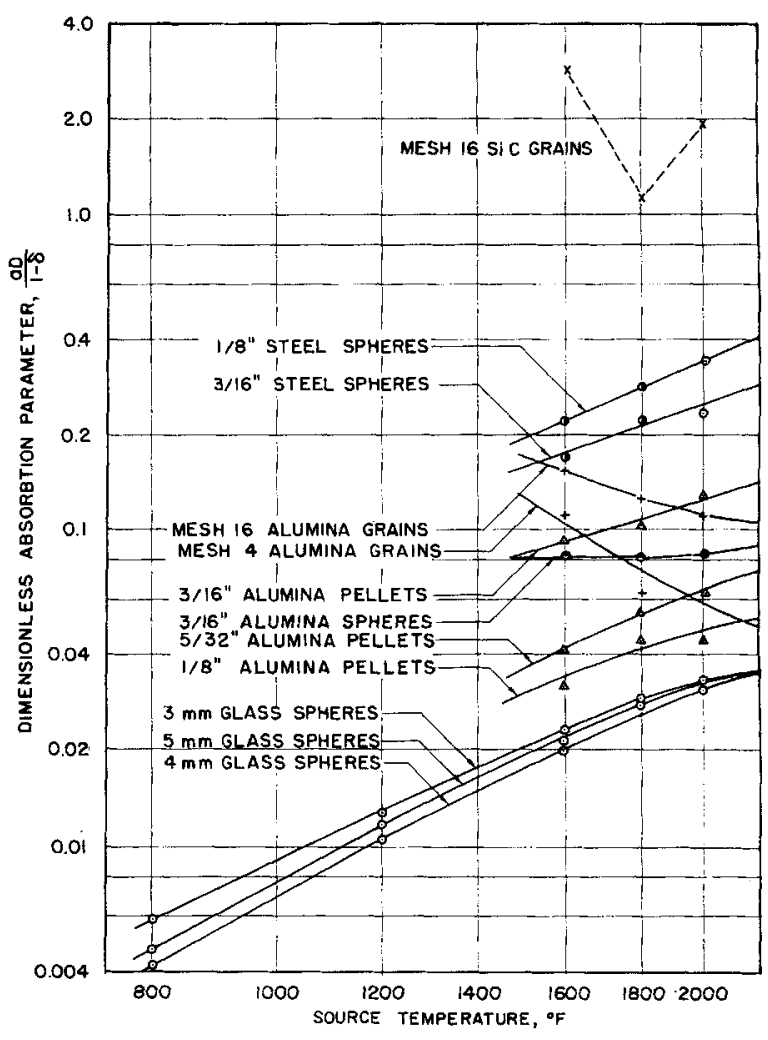

Fig. 8. Absorption parameter for packed beds. 
Table 2. Attenuation Cross Sections and Radiant Conductivity

\begin{tabular}{|c|c|c|c|c|}
\hline $\begin{array}{l}\text { Packing } \\
\text { particle }\end{array}$ & $\begin{array}{l}\text { Source } \\
\text { temper- } \\
\text { ature, }{ }^{\circ} \mathrm{F} \text {. }\end{array}$ & $\begin{array}{c}a_{,} \\
\left(\mathrm{ft}^{\prime}\right)^{-1}\end{array}$ & $\begin{array}{c}b \\
(\mathrm{ft} .)^{-1}\end{array}$ & $\begin{array}{c}k_{r} \\
\text { B.t.u. } /(\mathrm{hr} .) \\
\text { (ft. })\left({ }^{\circ} \mathrm{F} .\right)\end{array}$ \\
\hline GS-3 & $\begin{array}{r}800 \\
1,200 \\
1,600 \\
1,800 \\
2,000\end{array}$ & $\begin{array}{l}0.390 \\
0.843 \\
1.54 \\
1.88 \\
2.21\end{array}$ & $\begin{array}{l}977 \\
372 \\
190 \\
153 \\
129\end{array}$ & $\begin{array}{l}0.0140 \\
0.0840 \\
0.314 \\
0.515 \\
0.785\end{array}$ \\
\hline GS-4 & $\begin{array}{r}800 \\
1,200 \\
1,600 \\
1,800 \\
2,000\end{array}$ & $\begin{array}{l}0.209 \\
0.521 \\
0.988 \\
1.33 \\
1.59\end{array}$ & $\begin{array}{r}878 \\
298 \\
149 \\
114 \\
95.0\end{array}$ & $\begin{array}{l}0.0156 \\
0.105 \\
0.401 \\
0.689 \\
1.06\end{array}$ \\
\hline GS-5 & $\begin{array}{r}800 \\
1,200 \\
1,600 \\
1,800 \\
2,000\end{array}$ & $\begin{array}{l}0.178 \\
0.437 \\
0.812 \\
1.09 \\
1.24\end{array}$ & $\begin{array}{c}952 \\
296 \\
151 \\
114 \\
96.8\end{array}$ & $\begin{array}{l}0.0144 \\
0.106 \\
0.401 \\
0.689 \\
1.06\end{array}$ \\
\hline AS-3/16 & $\begin{array}{l}1,600 \\
1,800 \\
2,000\end{array}$ & $\begin{array}{l}2.74 \\
2.70 \\
2.84\end{array}$ & $\begin{array}{l}496 \\
483 \\
449\end{array}$ & $\begin{array}{l}0.120 \\
0.163 \\
0.227\end{array}$ \\
\hline $\mathrm{AP}-1 / 8$ & $\begin{array}{l}1,600 \\
1,800 \\
2,000\end{array}$ & $\begin{array}{l}1.06 \\
1.49 \\
1.47\end{array}$ & $\begin{array}{r}1,190 \\
860 \\
847\end{array}$ & $\begin{array}{l}0.0504 \\
0.0918 \\
0.120\end{array}$ \\
\hline $\mathrm{AP}-5 / 32$ & $\begin{array}{l}1,600 \\
1,800 \\
2,000\end{array}$ & $\begin{array}{l}1.00 \\
1.32 \\
1.54\end{array}$ & $\begin{array}{l}906 \\
682 \\
576\end{array}$ & $\begin{array}{l}0.0661 \\
0.116 \\
0.177\end{array}$ \\
\hline $\mathrm{AP}-3 / 16$ & $\begin{array}{l}1,600 \\
1,800 \\
2,000\end{array}$ & $\begin{array}{l}1.96 \\
2.18 \\
2.77\end{array}$ & $\begin{array}{l}682 \\
614 \\
496\end{array}$ & $\begin{array}{l}0.0877 \\
0.129 \\
0.205\end{array}$ \\
\hline$A G-16$ & $\begin{array}{l}1,600 \\
1,800 \\
2,000\end{array}$ & $\begin{array}{c}12.7 \\
10.6 \\
9.37\end{array}$ & $\begin{array}{l}779 \\
811 \\
837\end{array}$ & $\begin{array}{l}0.0762 \\
0.0968 \\
0.121\end{array}$ \\
\hline $\mathrm{AG}-4$ & $\begin{array}{l}1,600 \\
1,800 \\
2,000\end{array}$ & $\begin{array}{l}2.05 \\
1.16 \\
1.20\end{array}$ & $\begin{array}{r}789 \\
1,090 \\
1,010\end{array}$ & $\begin{array}{l}0.0758 \\
0.0729 \\
0.101\end{array}$ \\
\hline CG-16 & $\begin{array}{l}1,600 \\
1,800 \\
2,000\end{array}$ & $\begin{array}{l}272 \\
107 \\
192\end{array}$ & $\begin{array}{r}420 \\
1,050 \\
627\end{array}$ & $\begin{array}{l}0.108 \\
0.0714 \\
0.141\end{array}$ \\
\hline $\mathrm{SS}-1 / 8$ & $\begin{array}{l}1,600 \\
1,800 \\
2,000\end{array}$ & $\begin{array}{l}13.1 \\
17.0 \\
20.5\end{array}$ & $\begin{array}{l}450 \\
387 \\
343\end{array}$ & $\begin{array}{l}0.131 \\
0.200 \\
0.289\end{array}$ \\
\hline $\mathrm{SS}-3 / 16$ & $\begin{array}{l}1,600 \\
1,800 \\
2,000\end{array}$ & $\begin{array}{l}6.66 \\
8.70 \\
9.26\end{array}$ & $\begin{array}{l}370 \\
322 \\
314\end{array}$ & $\begin{array}{l}0.161 \\
0.243 \\
0.320\end{array}$ \\
\hline
\end{tabular}

tures above $1,500^{\circ} \mathrm{F}$, due to the reduced transmission.

Representative plots of the normalized transmission as a function of packed height are presented in Figures 2 and 3 . The two cross sections $a$ and $b$ in Equation (33) were evaluated for each packing and temperature by least-squares regression. The curves represent the resulting correlation. The standard deviations averaged $2 \%$ for the packings of glass spheres and $8 \%$ for the packings of silicon carbide grains. The greater standard deviations for the silicon carbide packing are due to the lower transmission and hence greater experimental uncertainty.

The transmission curves for packings of materials with a high absorptivity, such as silicon carbide, have steep

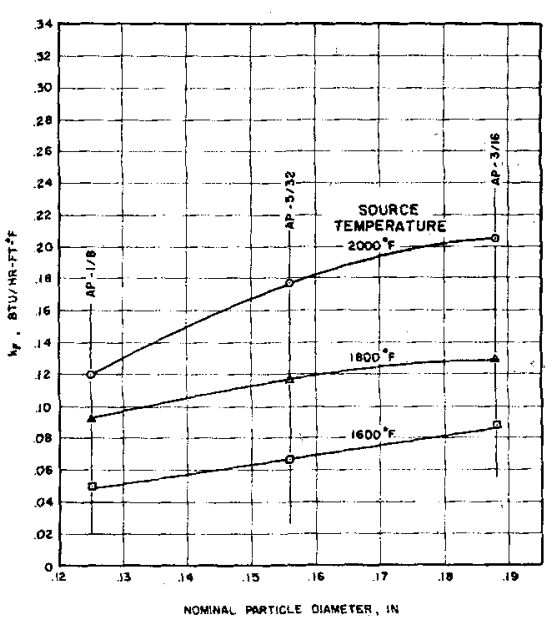

Fig. 9. Effect of particle size on radiant conductivity. slopes and are nearly straight on a semilogarithmic plot. The deviation of the transmission curves from a straight line, as for the glass packing, is due to back scattering. The variation in the transmission with temperature for the glass packings is consistent with the known variation in the transmissivity of glass with wave length. Glass is quite opaque at $4.9 \mu$, which is the wave length of maximum intensity for an $800^{\circ} \mathrm{F}$. source, but is quite transparent at $2.2 \mu$, which is the wave length of maximum intensity for a $2,000^{\circ} \mathrm{F}$. source.

The character of the transmission curves, the scatter in the data, and the degree of correlation for all of the other materials tested were intermediate to the illustrated results for glass spheres and silicon carbide grains.

\section{ABSORPTION AND BACK SCATTERING CROSS SECTIONS}

The computed absorption and back scattering cross sections per unit volume of packed bed for all of the packings and source temperatures are given in Table 2. The relative importance of absorption and back scattering is evident from this tabulation. Back scattering is the primary mechanism of attenuation of radiation for all of the packings. The back scattering cross sections are of the same order of magnitude for all of the materials tested, but the absorption cross sections have a wide range of values. The ratio of the back scattering to the absorption cross section is greater than 50 for the aluminum oxide and glass packings, varies from 17 to 50 for the steel packings, and from 1.5 to 10 for the silicon carbide packing.

The cross sections for packings of glass spheres and steel spheres are plotted vs. source temperature in Figures 4 and 5. $a$ increases and $b$ decreases sharply with increasing source temperature for the glass spheres. The same trends but weaker dependence were found for the steel spheres and for the other regularly shaped packings. The granular packings demonstrated slight and irregular dependence on source temperature.

The cross sections are presumed to be closely related to the absorptivities of the materials of which the packings are composed. These absorptivities and the cross sections are in general a function of both the spectral distribution of the incident radiation (the source temperature) and the temperature of the material. However Hottel (16) states that the total absorptivity of nonmetals varies more with the former than with the latter. For metals the temperature of the material may also have a significant effect on the total absorptivity. Some uncertainty 


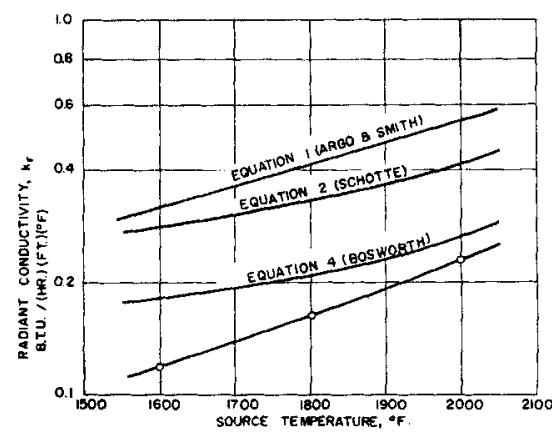

Fig. 10. Comparison of derived and predicted volues for radiant conductivity of packed beds of 3/16-in. alumino spheres (AS-3/16).

therefore exists regarding the applicability of the cross sections presented herein for the steel spheres insofar as the absorptivity changes with the temperature of the steel.

The cross sections of aluminum oxide, steel, and silicon carbide packings at several source temperatures are plotted vs. the nominal emissivity of the materials for the same temperatures in Figure 6. The absorption cross sections generally increase sharply with emissivity, but the back scattering cross sections decrease.

Since $a$ and $b$ would be expected to be proportional directly to the total projected area of the particles per unit volume of packing, the dimensionless groups $b D /(1-\delta)$ and $a D /(1-\delta)$ are plotted vs. source temperature in Figures 7 and 8 . Until additional data are obtained to elucidate further the dependence on diameter, shape, porosity, emissivity, transmissivity, and temperature Figures 6,7 , and 8 are recommended for interpolation and extrapolation. Owing to the high degree of uncertainty in the transmission measurements for the silicon carbide grains, the indicated maximum in $b$ and minimum in $a$ are presumed to be without significance.

\section{RADIANT CONDUCTIVITY}

The radiant conductivity is a less fundamental quantity than the cross sections but provides an indication of the relative contribution of radiant transfer and is directly applicable for process calculations. The internal radiant conductivity was calculated for each packing and condition from Equation (24). The resulting values are included in Table 2 and in Figures 4 and 5 . The effects of particle size is further illustrated in Figure 9 for the packings of aluminum oxide pellets. Several trends are evident from the table and figures. $k_{r}$ increases with temperature for all of the packings and with particle size except for the irregular grains of aluminum oxide. For a given particle size the radiant conductivity appears to increase in the order:

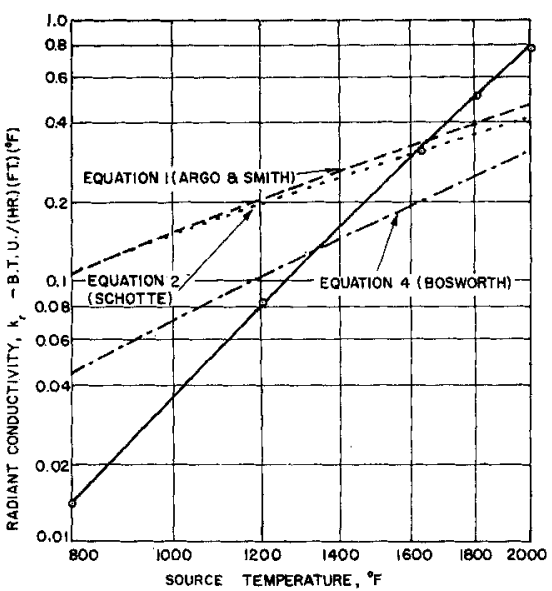

Fig. 11. Comparison of derived and predicted values for radiant conductivity of packed beds of 3-mm. glass spheres (GS-3).

aluminum oxide, steel, silicon carbide, and glass. The low radiant conductivity for the aluminum oxide packings is attributable to the high reflectivity of the particles. The greater emissivity of the silicon carbide and steel particles results in a higher radiant conductivity. On the other hand the still higher values for glass packings are due to direct transmission of low wave length radiation through the glass rather than to absorption and emission.

Previous theoretical predictions of the radiant conductivity are compared with the derived values for $3 / 16$-in. alumina spheres in Figure 10 and for $3-\mathrm{mm}$. glass spheres in Figure 11. All three predictions are higher than the derived values for the alumina spheres but provide a reasonable estimate of the effect of temperature. A similar result was obtained for the other alumina and silicon carbide packings. For the glass packings the predictions are all higher than the derived values at low temperatures and lower at high temperatures. The latter effect can be explained in terms of the transmissivity of glass for low wave length radiation. As was pointed out by Schotte (4) previous theories were limited to opaque packings.

There is considerable uncertainty concerning the emissivity of the steel spheres at the test conditions owing to the aforementioned dependence on packing temperature and also to the presence of a slight oxide coating. For an estimated emissivity of 0.4 all three theoretical predictions are also too high for the steel spheres. If the emissivity were as low as 0.2 , the prediction of Schotte would be very good, the prediction of Bosworth would still be high, and the prediction of Argo and Smith would be too low.

The models, including the one utilized in this investigation, all have the form

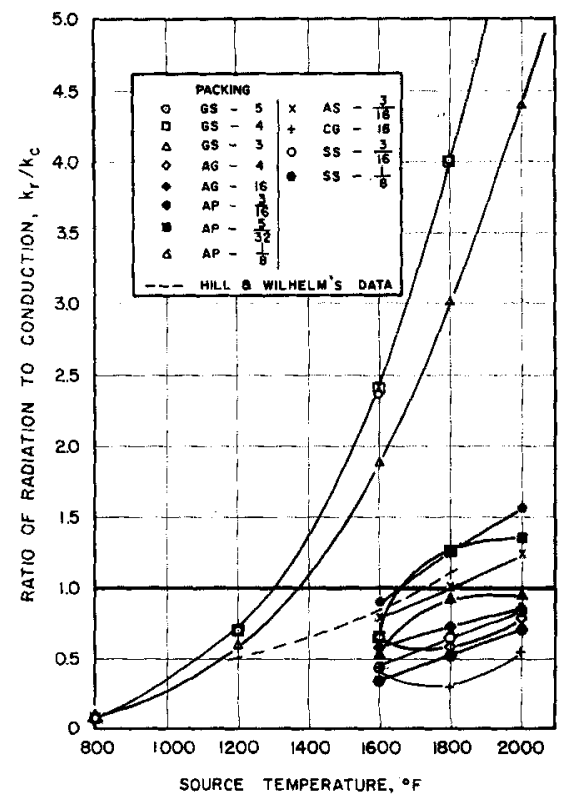

Fig. 12. Radiant contribution to heat transfer in packed beds.

$k_{r}=4 F \sigma D T^{3}$

with the following expressions for $F$ :

Equation no. $\quad F$

Argo and

Smith (3)

Schotte (4)

Bosworth (9)

$\epsilon /(2-\epsilon)$

This investi-

gation

The dependence of $a$ and $b$ and hence $k_{r}$ on emissivity is observed in Figure 6 to be more complex than predicted by Argo and Smith, Schotte, or Bosworth. None of these three expressions indicate any dependence of $F$ on particle shape, particle size, or transmissivity, although the data demonstrate a dependence on all of these variables. The expressions of Argo and Smith and Schotte imply a dependence on temperature but only insofar as $\epsilon$ is dependent on temperature.

\section{RATIO OF RADIATION TO CONDUCTION}

The ratio of heat transfer by radiation to heat transfer by conduction is of direct practical interest. This ratio is plotted for all of the experimental packings in Figure 12. The correlation of Wilhelm, Johnson, Wynkoop, and Collier (14) was used to estimate the values of $k_{c}$. As pointed out by Hill and Wilhelm (7) this correlation was based on data for overall conductivities including wall effects and is not strictly comparable to the data herein for radiant conductivities far from a wall. The computed ratios should nevertheless be informative and semiquantitative. 
The ratios for glass packings are seen to be the largest and to have the greatest sensitivity to temperature. For the 4- and 5-mm. glass spheres radiation accounts for $50 \%$ of the total heat transfer at $1,300^{\circ} \mathrm{F}$. and $85 \%$ at $2,000^{\circ} \mathrm{F}$. The opaque particles are observed to have lower ratios which are also less sensitive to temperature. However at $2,000^{\circ} \mathrm{F}$. the radiant contribution is $33 \%$ even for the silicon carbide grains. This ratio has previously been determined experimentally only for Hill and Wilhelm's 3.8-mm, aluminum oxide spheres (7). A curve representing their data is included in Figure 12. Their data agree well with the data of this investigation for $3 / 16$ in. (4.8-mm.) aluminum oxide spheres. The radiation-to-conduction ratio is observed to increase with particle size except for the irregular aluminum oxide grains. The effect of particle size is most pronounced at high temperatures.

\section{APPLICATION OF RESULTS}

The experimental values of the absorption and back scattering cross sections are directly applicable in Equations (6), (7), and (8) for heat transfer through a slab of packing. Under most circumstances approximate solutions such as Equaitons (14) and (15), which imply temperature-mean values of $a$ and $b$ as well as the assumptions noted, are satisfactory. For glass and aluminum oxide packings, and even steel packings, absorption was observed to be negligible, and still simpler solutions such as Equations (26) and (27) are applicable.

For optically thick packings the internal radiant conductivity can be utilized directly and heat transfer in packed beds represented as a simple conductive process with $k_{t}=k_{r}+k_{r}$. Although the direct use of $a$ and $b$ is limited to one-dimensional heat transfer through a slab of packing, this total conductivity can be used directly in the appropriate differential energy balance (and solution) for any geometry and application of a packed bed without fluid flow.

\section{SUMMARY AND CONCLUSIONS}

The experimental data for radiant transmission through packed beds were correlated very well in terms of the two-flux model, thereby yielding values of the effective absorption and back scattering cross sections per unit volume of packed bed. An effective conductivity for radiation can in turn be calculated from these quantities.

Back scattering was found to be the major mechanism for attenuation of radiation for all packings. Absorption was found to be significant only for the packings of silicon carbide grains. Packings of glass spheres have the greatest radiant conductivity because of their transmissivity and packings of aluminum oxide the least because of their high reflectivity. In general the radiant conductivity increases with temperature, particle size, and emissivity. Radiation becomes important relative to conduction above $1,600^{\circ} \mathrm{F}$. for all of the materials tested.

The expressions previously proposed for the a priori prediction of the radiant conductivity do not always indicate the correct dependence on temperature and the other variables owing to their failure to take transmissivity and scattering into account correctly. Hill and Wilhelm (7) did propose expres. sions which account for scattering and transmissivity, but their equations do not permit the a priori estimation of radiant conductivity since a number of the constants in their expression must be determined experimenatlly.

The data of this investigation did not cover a sufficient range of conditions and properties to permit development of a generalized correlation for all packed beds but do provide a basis for interpolation and moderate extrapolation. The results are directly applicable in any process involving heat transfer in a packed bed without fluid flow.

\section{ACKNOWLEDGMENT}

The loan of experimental equipment by the Owens-Corning Fiberglas Corporation is greatly appreciated. J. C. Chen received fellowship support from the Monsanto Chemical Company: The suggestions of Professor C. M. Chu were invaluable.

\section{NOTATION}

$a=$ absorption cross section per unit volume of packing, $(\mathrm{ft} .)^{-1}$

$b=$ back scattering cross section per unit volume of packing, $(\mathrm{ft} .)^{-1}$

$D=$ diameter of particle, ft. (except as noted)

$F \quad=$ function in Equation (34)

$h=$ height of packing in experiments, ft.

$i=$ radiant heat flux density in forward direction, (B.t.u.)/ (hr.) (sq.ft.)

$i_{o} \quad=$ incident heat flux density from source, (B.t.u.) /(hr.) (sq.ft.)

$j=$ radiant heat flux density in backward direction, (B.t.u.)/ (hr.) (sq.ft.)

$k=$ thermal conductivity, (B.t.u.)/ (hr.) (ft.) $\left({ }^{\circ} \mathbf{F}\right.$.)

j = mean free path for radiation, $\mathrm{ft}$.

$L=$ thickness of packed bed

$n=$ index of refraction of solid relative to fluid $q$ = heat flux density, (B.t.u.)/ (hr.) (sq.ft.)

$s=$ scattering cross section per unit volume [Equation (5)], $(\mathrm{ft} .)^{-1}$

$S_{n}=$ normalized transmission [Equation (33)]

$T=$ temperature, ${ }^{\circ} \mathbf{R}$.

$x=$ distance through packing, $\mathrm{ft}$.

$\delta=$ void fraction of packing

$\epsilon \quad=$ emissivity

$\boldsymbol{\sigma}=$ Stefan-Boltzmann constant $=$ $1.73 \times 10^{-3} \quad$ (B.t.u.) $/($ hr. $)$ (sq.ft.) $\left({ }^{\circ} \text { R. }\right)^{4}$

\section{Subscripts and Superscripts}

$c \quad=$ conduction

$g=$ gas phase

$r \quad=$ radiation

$s=$ solid phase

$t=$ total (radiation plus conduction)

$1=$ boundary plane of packing at $\boldsymbol{x}=0$

$2=$ boundary plane of packing at $x=L$

$0=$ mean temperature

\section{LITERATURE CITED}

1. Nusselt, W., Z. bayer. Revisions-ver., No. 13 and 14 , p. 85 (1913)

2. Damköhler, G., "Der Chemie-Ingenieur," Vol. 3, Part 1, p. 445, Eucken-Jakob, Akademische Verlagegesellschaft M.B.H., Leipzig, Germany (1937).

3. Argo, W. B., and J. M. Smith, Chem. Eng. Progr, 49, 443 (1953).

4. Schotte, William, A.I.Ch.E. Journal, 6, 63 (1960).

5. Laubitz, M. J., Can. J. Phys., 37, 798 (1959).

6. Yagi, Sakae, and Daizo Kunii, A.I.Ch.E. Journal, 3, 373 (1957).

7. Hill, F. B., and R. H. Wilhelm, ibid., 5, 486 (1959).

8. Rosseland, S., Theoretical Astrophysics," Oxford Univ. Press, Clarendon, England (1936).

9. Bosworth, R. C. L., "Heat Transfer Phenomena," Wiley, New York (1952).

10. Van der Held, E. F. M., Appl. Sci. Research, A3, 237 (1952); A4, 77 (1953).

11. Hamaker, H. C., Philips Research Repts., 2, 55, 103, 112, 420 (1947).

12. Gorring, R. L., and S. W. Churchill, Chem. Eng. Progr., 57, No. 7. p. 53 (July, 1961).

13. Larkin, B. K., and S. W. Churchill, A.I.Ch.E. Journal, 5, 467 (1959).

14. Wilhelm, R. H., W. L. Johnson, R. Wynkoop, and D. W. Collier, Chem. Eng. Progr., 44, 105 (1948).

15. Chen, J. C., Ph.D. thesis, Univ. Michigan, Ann Arbor, Michigan (1961).

16. McAdams, W. H., "Heat Transmission," 3 ed., McGraw-Hill, New York (1954).

17. Norton, F. H., "Refractories," 3 ed., McGraw Hill, New York (1949).

Manuscript received May 8, 1961; revision received June 8, 1962; paper accepted June 13 1962. Paper presented at A.Y.Ch.E. Cleveland meeting. 